\title{
Einleitung: Zur Methodik mit Blick auf die Variationen des Prometheus-Mythos
}

In jener Gegend reist man jetzt nicht gut;

Und hast du Geist, sei doppelt auf der Hut!

Man lockt und liebt dich, bis man dich zerreisst,

Schwarmgeister sind's - da fehlt es stets an Geist! (Friedrich Nietzsche, Vorsicht (Gedicht Nr. 37 in: Werke in sechs Bänden, hrsg. von Karl Schlechta, München 1980, Bd. III, S. 25.))

Das hier vorgelegte Buch traktiert Franz Kafka als den „Blutsbruder“ des mesmeristisch interessierten Heinrich von Kleist, der der Prager eigener, entschiedener Aussage nach gewesen ist, als auch einen ,electrischen Prometheus“. Es versteht sich dabei als ein Buch für Hermeneutiker, vulgo für Schlüsselbesitzer, vor allem solche, die ebenfalls eine „Dritte Kultur“" anstreben, und nicht als eines für „Dietriche“, wie sie in Nietzsches anderen Versen gemeint sind: „,Mögen alle Schlüssel doch/Flugs verloren gehen/Und in jedem Schlüsselloch/ Sich ein Dietrich drehen!'/Also denkt zu jeder Frist/Jeder, der ein - Dietrich ist". ${ }^{\text {" }}$ Das ist das eine: Zugang durch die Originalschlüssel; eben durch ein Zusammenschauen der beiden Kulturen bewirkt, wie sie als einander ausschliessende ja erst seit dem Positivismus (und vor allem in Europa) existier(t)en. Das andere ist: Der „electrische Prometheus“ Franz Kafka, jener expressionistische, jüdische,

\footnotetext{
${ }^{1}$ Friedrich Nietzsche, Fromme Wünsche, Vorspiel zur Fröhlichen Wissenschaft, Nr. 51, bei Schlechta a. a. O., Bd. III, S. 29.

(C) Der/die Autor(en) 2021

B. Neumann, Umrisse einer Dritten Kultur im interdisziplinären Zusammenspiel zwischen Literatur und Naturwissenschaft, ELECTRISCHER PROMETHEUS. Umrisse einer Dritten Kultur im interdisziplinären Zusammenspiel zwischen Literatur und Naturwissenschaft, https://doi.org/10.1007/978-3-662-63204-8_1
} 
deutsch-assimilierte, aber eben auch „altösterreichische“ Romancier aus Prag, wäre dann das genaue Gegenteil dessen, was ein anderer Österreicher, Adolf Hitler nämlich, als seinen „Prometheus“ angesehen hat - den (deutschen) „Arier“ als den Prometheus der Menschheit, als eine Mythengestalt, die dieser das Feuer politisch richtiger Erkenntnis bringen sollte. Darunter machte der „Führer“ es nicht, auch er auf seine Weise ein "Schwarmgeist“. Wir dagegen statuieren einen „electrischen Prometheus“ Kafka und dessen „,kafkaeskes“ und darum elektrisierendes Erzählen - mit aller gebotenen Nüchternheit und gegen vergangene wie gegenwärtige Schwarmgeister gerichtet.

Dass und wie der Prager dies gewesen ist, soll vor allem durch eine ,dichte Beschreibung“ (des bekannten Ethnologen Clifford Geerts „Thick Description“ gibt dabei eine theoretische Richtschnur ab) ${ }^{2}$ von Kafkas Beziehung zu seinem „Blutsbruder“ Heinrich von Kleist samt dessen galvanisch-elektrischem Verfallensein sinnfällig gemacht werden, - was schon in sich ein desideratum darstellt, hat doch die Forschung zum Verhältnis Kafka/Kleist insgesamt keine besondere Priorität erfahren (trotz hervorstechender Einzelarbeiten, etwa von P.-A. Alt). Da der mathematische Beweis in der Literatur- wie Kulturwissenschaft nicht angetreten werden kann, muss es, wie erwähnt im Sinne des Ethnologen Geertz, vor allem aber in dem noch näher darzustellenden von Stephen Greenblatts New Historicism, darum gehen, eine so grosse Anzahl von ,selbst sprechende" Textmaterialien zusammenzutragen, dass diese am Ende zur Evidenz sich zusammen schliessen, bzw. zusammenschiessen - so wie eine Flüssigkeit im chemischen Prozess ihren Aggregatzustand verändern, eine andere, höhere Wertigkeit und intensivierte Konzentriertheit zu erlangen vermag. Dabei wird vor allem die Metapher, vom Griechen Aristoteles bis hin zum Münsteraner Hans Blumenberg mit ganz grossen Erkenntnisaufgaben betraut, uns hilfreich beispringen müssen; gerade um den hier angestrebten paradigmatischen Transfer von naturwissenschaftlicher, vor allem physikalisch-astronomischer Erkenntnis in das Medium der Literatur mit ihren verschiedenen Genres zu ermöglichen. Die Metapher soll es an den Tag bringen - etwa die zentrale des Hauses, der Höhle, des in der Kinderwelt noch geschlossenen Kosmos' im Falle von Kafka, der sich einmal, aus Anlass der Betrachtung eines Kinderphotos, der Knabe steht verloren und verlassen inmitten der arrangierten Kulissenwelt der frühen Photographie, als einen „Ruinenbewohner“, also als exemplarisch „unbehaust“, empfunden hat.

\footnotetext{
${ }^{2}$ Vor allem in: Clifford Geertz, The Interpretation of Cultures. Selected Essays, New York 1993 (Basis Books).
} 
Was darin sich aussprach, versteht buchstäblich nur, wer, wie immer verwegenungenau auch, versuchen wollte, die Befindlichkeitsgeschichte des Menschen in groben Zügen nachzuempfinden, sie ,,anzudenken“. Hier existieren eindrucksvolle Vorläufer wie auch Vorbilder. Immer schon, nicht erst zunehmend systematisch mit dem griechischen Denken, das bei Aristoteles dann sogar fähig erschien, die Umwandlung von Energie in Materie und vice versa zu denken (was wiederum kein anderer als Werner Heisenberg als Vater der „Unschärferelation“ festgeschrieben hat, in Physik und Philosophie, einem Buch, von dem später noch ausführlich die Rede gehen wird), schon immer also wurde der geregelt-feste Sternen- und Planetenlauf, wurden Sonne und Mond in ihren fixen Umlaufbahnen in Anspruch genommen für die Vernünftigkeit und damit „Behausbarkeit“ der Welt, für unsere blau-grüne Erde als einen Ort gesicherten Lebens. Bekanntlich hatte das alles begonnen mit der beruhigenden, sinnstiftenden Kosmos-Schau der Griechen, von Demokrit und Thales bis hin zu Aristoteles, und hatte sich nach dem Mittelalter über Galilei und Kepler fortgesetzt in des Philosophen Leibniz' Lehre von der ,prästabilisierten Harmonie“ des da noch Newton'schen Kosmos'. War dann aber auch als Entgegensetzung zugegen in der Ablehnung der Frühromantik gegenüber der geraden Linie, insgesamt gegen das Gerade und Abgezirkelte: „So werden für die Romantiker der geometrisierte Raum und die geometrisierte Zeit zum Schreckbild einer schlechten Aufklärung“. 3 Eine Schreckvorstellung, die dann bei Einstein in eine erkenntnisstiftende umschlägt, wenn er die Kategorien Raum und Zeit nicht mehr unabhängig voneinander denkt. Das hatte auf seine Weise bereits beider Vorläufer, der pessimistische und ,asiatisch“ orientierte Philosoph Schopenhauer, getan. Und damit seinerseits ein Ende der ,positivistischen“ Aufklärung samt dem Beginn des Siegeszugs einer nunmehr skeptischen Moderne auch in Philosophie und Literatur markiert, wie sie sich dann in Nietzsche, Hofmannsthal und eben auch Kafka vollenden sollte. Grundlegend war dafür die erwähnte Einstein'sche Postulierung der Abhängigkeit von Raum und Zeit voneinander gewesen, die sich danach im elektronenmikroskopischen Blick ,nach innen“, eben auf die verwirrend-bedrohliche Sprunghaftigkeit der elektromagnetisch bestimmten Partikelbahnen um den Atomkern herum, quantentheoretisch fortsetze. Alles gipfelte schliesslich in der verheerenden Sprengkraft dessen, was der griechische Begriff „Atom“ eigentlich ausgeschlossen hatte: In der Aufsprengung, der Spaltung des „Unteilbaren“, eben

\footnotetext{
${ }^{3}$ Rüdiger Safranski, Romantik. Eine deutsche Affäre, München 2007 (Hanser), hier wie im Folgenden zitiert nach der neuen Sonderausgabe als Fischer Taschenbuch, Frankfurt/Main, März 2020, S. 307.
} 
des Uran-Atomkerns zuerst durch Otto Hahn. Was danach zum Albtraum des Atombomben-Einsatzes am Ende des Zweiten Weltkrieg führte, - zu einem Albtraum, der in seiner entgegengesetzten Form des „Schnellen Brüters“, so dieser sich technisch realisieren liesse, energiepolitisch nicht weniger als den Himmel auf Erden bedeuten würde. Die Anwendung der Atombombe, deren Konstruktion ohne Einsteins Theorien nicht möglich gewesen wäre, brachte den Autor der Physiker Friedrich Dürrenmatt dazu, die Ordnung der Literaturgattungen selbst zu verändern, den Tod der Tragödie zu verkünden, wovon noch ausführlicher zu handeln sein wird. Die Kahlschlag-Komödien des Schweizer Autors lebten ja immer vom Bankrott des - in sich ja optimistisch-beruhigenden - Newtonschen Weltbildes, wie immer in seinen Physikern Newton in Einstein stecken sollte und umgekehrt. Der allerneueste Schrecken aber wird darin erkennbar, dass das bucklige Fräulein Doktor als ihrerseits die Gefangenwärterin der beiden irren Physiker (die eigentlich ganz zweckrational denkende Geheimdienst-Agenten sind), aus heutiger Wahrnehmung doch tatsächlich als die aus dem Ruder gelaufene Chefs von Facebook oder auch Amazon erscheinen könnten? Das setzte dann voraus, dass der Philosoph Markus Gabriel in seinem neuesten Buch Moralischer Fortschritt in dunklen Zeiten einen entscheidenden Punkt getroffen hätte, wenn er ausführte: „Ja, die sozialen Medien haben wesentlich zu dem (gegenwärtigen, „fake news" legitimierenden, B.N.) Trump-Problem beigetragen. Ich halte sie für Maschinen, die in ihrem Wesen zur Unterminierung der liberalen Demokratie und der Aufklärung führen. Facebook insbesondere ist eine gefährliche postmoderne Maschine, die zur Zerstörung des Werts der Wahrheit beiträgt. Mark Zuckerberg sagt ja selbst, Facebook sei nicht zuständig für Wahrheitsfindung. ... Auch die

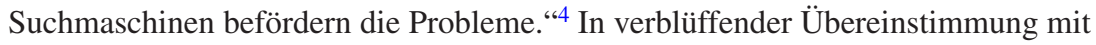
dieser so ganz aktuellen Problemstellung übernahm bereits beim hellsichtigen Dürrenmatt ein bucklichtes Fräulein die Abwicklung einer ehemals vernunftbegabten bürgerlichen Welt, und das gegen den Einspruch des marxistischen Autors Bertolt Brecht, dessen Galileo bei aller Skepsis noch einmal die progressive Welt der neu-griechischen Renaissance beschwor, in der der Held dem ihm klerikal drohenden Scheiterhaufen noch listig auszuweichen vermag.

\footnotetext{
${ }^{4}$ Interview mit Markus Gabriel in: Der Spiegel, Nr. 32, 1. 8. 2020, anlässlich des Erscheinens seines oben genannten Buchs 2020 im Ullstein Verlag. Gabriels Aussagen, „optimistisch“, wie sie nach eigenem Verständnis sind, markieren einen entschiedenen Fokus in vielen Diskussionen des Herbstes im Jahr 2020, und sie verdanken ihre Aktualität u. a. der von ihm gewählten Verschränkung der Corona-Krise mit dem globalen politischen Klima.
} 
Einstein dann war es vorbehalten, in seiner verblüffend offenherzigen, bislang quasi unbekannten Aussage zu Max Brods Galilei das Kaleidoskop der Physikerund Dichterstellungnahmen noch ein entscheidendes Stück weiter zu drehen. Hinzu kommt: Nach Deutungen einschlägiger Zeitgenossen, Heinz Ludwig Arnolds etwa, soll auch bei Dürrenmatt der Raum keine unabhängige Grösse mehr darstellen, sondern seinerseits kategorisch von seiner Abhängigkeit von der Zeit begrenzt erscheinen. Andererseits hat Dürrenmatt, der ja ein literaturtheoretisch sehr interessierter Autor gewesen ist, geschrieben, dass nur die hergebrachte aristotelische Einheit von Raum, Zeit und Handlung seinen avancierten Physikern gerecht zu werden vermöchte. Die völlig selbstverständlich zur Schau gestellte Widersprüchlichkeit des Dramatikers Dürrenmatt machte einen wesentlichen Teil seiner Faszination aus. Und doch ist bei dem Schweizer der Ausgangspunkt immer sein aus allen rationalen Angeln gehobenes, vormals so idyllisches Schweizertal, wo er als Bub noch fraglos zu Hause gewesen war: Das eidgenössische Gegenstück zum Thales in Hans Blumenbergs Das Lachen der Thrakerin. Die radikale Wende hin zur allein noch gültigen Komödie oder sogar Farce erscheint bei Dürrenmatt stets verschwistert mit den Entdeckungen der allerneuesten Physik. Dass es keine Helden mehr gäbe, keine geschlossenen Charaktere, keine solide tragische Handlung mehr und keinen Sinn im Opfertod: Das alles löst, wie der Klappentext der Physiker es festschreibt, die ,eindimensionale, auf cartesianischer Denksystematik beruhende" alte Welt der noch prä-Einsteinischen Sicherheiten auf. Solche Umwälzung entspricht schon der Einstein/Heisenbergschen Revolution des physikalischen Weltbildes. Gewiss: Der strikten „Auflösung der Einheit von Raum, Zeit und Handlung, die unter Verrückten spielt, kommt nur noch die klassische Form bei. " ${ }^{5}$ Doch diese Physiker „verrücken“ nur deshalb alles, weil Dürrenmatt, der entlaufene Expressionist (der er als Maler bis zuletzt geblieben ist), dem Schrecken der jeweils neuesten Physik begegnet war - so wie vor ihm bereits Wilhelm Dilthey in Das Erlebnis und die Dichtung, einer Schrift u. a. über die Frühromantiker. Fast ein Jahrhundert vor dem Schweizer hatte bereits Wilhelm Dilthey an Luise Scholz, die ihm vertraute Frau seines guten Freundes Joachim Scholz, das folgende geschrieben (eingebettet in seine Eindrücke nach intensiver Kleist-Lektüre, welche ihn offenbar in die richtige Stimmung versetzt hatte): „...die Sterne vom Himmel gefallen und die Sonne von dem furchtbaren Fenriswolf verschlungen. ... und die tückischen

\footnotetext{
${ }^{5}$ Karl Ludwig Arnold, Materialien zu Dürrenmatts ,Physiker', Schrödel Verlag, Dezember 2009, S. 12.
} 
und ungeschlachten Riesen herrschen ...“ Ferner: „Das Schreckliche soll von dem Menschen mit einem heiligen Vertrauen auf die göttliche Vernunft hingenommen werden: jene Stimmung ihm gegenüber aber ist heillos, dumpf und entsetzlich - entsetzlich wie der Gedanke von den zufällig kreisende Atomen in der Wissenschaft - und zugleich wie dieser ohne Grösse." 6 Bereits der Theoretiker der späteren "Geistesgeschichte" war also hervorragend orientiert über den Fortschritt in den Naturwissenschaften, und eben auch in der damals erst sich schemenhaft abzeichnenden Atomphysik, der bei ihm Panik geradezu, eine existentielle Angst um die klassischen Ideale der Winckelmann'schen „Einfalt“ samt „stiller Grösse“, auslöste. Bereits hier schien diesem wohl einflussreichsten Literaturtheoretiker des Wilhelminismus jener Meteor zu drohen, dessen Ankunft in Dürrenmatts ultimativem Schauspiel dann alles Leben verwüsten wird. Keine Spur mehr davon, dass, wie noch bei dem Romantiker Richard Wagner, die positive Gestalt eines neuen Prometheus auftreten könnte, eben Siegfried als Lichtbringer und neuer Christus in einem, zusammengeführt zu einer überaus positiven Figur. ${ }^{7}$ Ganz wie bei Kafka, und von den verschiedensten Deutern auch realisiert, rückt darum auch bei Dürrenmatt das Paradoxe ins Zentrum der Darstellung. Allein das ,paradoxale Gleichnis“ erweist sich noch als tragfähig, wie Karl Ludwig Arnold erkannt hat. Eben das teilt der Endzeit-Dramatiker Dürrenmatt mit dem revolutionären Epiker Kafka. Das ,,paradoxale Gleichnis“ ist beider Spezialität. In beiden Fällen sind Physiker die Verursacher solcher literarischen Besonderheit - wie immer diese nicht monokausal auf einen „Einfluss der Physik" zurückgeführt werden darf wie kann. Die hier vorgelegte Arbeit beansprucht denn auch keinesfalls, nun alles restlos kausal-,,materialistisch“ einsinnig begründen zu können, erst recht nicht, womöglich „,das Kafkaeske zu entschleiern." Dennoch meint sie, zu dessen besserem Verständnis Wichtiges beitragen zu können, eben im Bewusstsein, dass alles durch Monokausalität begrenztes Denken zu vermeiden sei. Eben diese Einsicht hat sie der sog. „Postmoderne" zu verdanken, ohne damit deren extreme Besonderheiten, jene „Dekonstruktion“ um jeden logischen Preis, die beispielsweise Hans Magnus Enzensberger ein „Kasperletheater“ zu nennen vermochte, zu teilen.

Und Franz Kafka stand nicht gänzlich allein, gerade auch nicht mit Blick auf die letztgeschilderte Problematik. Neben dem Prager war auch Robert Musil

\footnotetext{
${ }^{6}$ In: Heinrich von Kleists Nachruhm. Eine Wirkungsgeschichte in Dokumenten. Neu herausgegeben von Helmut Sembdner, München 1997, S. 296 f.

${ }^{7}$ Dazu R. Safranski, Romantik, a. a. O., S. 400.
} 
ein Autor des beginnenden gesellschaftlich-politischen Untergang „Kakaniens“ im ersten Jahrzehnt des 20. Jahrhunderts, mithin in Zeiten einer zugleich künstlerischen Hochblüte, gewesen. Musils Biograph Karl Corino hat eindrücklich herausgearbeitet, dass das anbrechende 20. Jahrhundert damals für jeden, der wirklich informiert war über seine Fachgrenzen hinaus, als eines der umwälzenden technisch-naturwissenschaftlichen Fortschritte empfunden werden musste. ${ }^{8}$ Jetzt erst endete wirklich ,das tausendjährige Reich der Romantik“, dessen Ende Heinrich Heine bereits am 3. Januar 1846 an Varnhagen von Ense vermeldet hatte, garniert mit folgender Selbsternennung: "...und ich selbst war sein letzter und abgedankter Fabelkönig“. Sein Atta Troll von 1841 sei der „Schwanengesang der untergehenden Periode gewesen“. Wir werden darauf zurückkommen (in der Abteilung B) anlässlich der jüngeren Novellentexte des Botho Strau $\beta$ als des vermutlich literarisch anspruchsvollsten „modernen Romantikers“, die eben deswegen in unseren Kontext gehören. Noch Schlimmeres aus Sicht dieser ,Nachtigallen“-Romantik sollte freilich, und noch zu Musils und eben auch Kafkas Zeiten, folgen: „Der Motorflug begann 1901 mit Whitehead bzw. 1903 mit den Gebrüdern Wright, das erste brauchbare Unterseeboot baute J. P. Holland 1898, die ersten grossen Alpentunnel waren die vom Mont Cenis (13 km lang, 1871 fertiggestellt) und vom Gotthard (15 km lang, 1882 fertiggestellt), den $20 \mathrm{~km}$ langen Simplon-Tunnel begann man just 1898; die ersten Drucktelegraphen bauten Hughes (1855) und Baudot (1874), die Geschichte des Telephons fing mit Reis (1861) und Bell (1876) an, 1877 erfand Edison den Phonographen,1895 entdeckte Röntgen die nach ihm benannten Strahlen, wofür er 1901 den ersten Nobelpreis für Physik erhielt. Kein Zweifel, es herrschte unter den Technikern, Ingenieuren, Physikern und Chemikern eine heute kaum noch vorstellbare Aufbruchsstimmung ..."9 Man lebte in einer umstürzend neuen Zeit, und Kafka war zudem als altösterreichischer Prager besonders sensibilisiert für derartige Geistes-Revolutionen, die im Schatten des Hradschin bis in Renaissance und Barock, mindestens, zurückreichten. War immer schon das Kind einer ,magischen“ Stadt, deren Wahlbürger dann auch Einstein zu werden wünschte, gewesen, was noch näher ausgeführt werden wird. Aufgewachsen in einer Verbindung zwischen „Erster" und „Zweiter Kultur“, die seinerzeit bewirkte, dass Musil auf eine Kritik Jakob Schaffners (in der viel gelesenen Neuen Rundschau) mit einer schon in sich ungewöhnlichen, und nun

\footnotetext{
${ }^{8}$ Karl Corino, Robert Musil. Eine Biographie, Reinbek 2003, S. 122.

${ }^{9}$ Karl Corino, a. a. O., S. 122.
} 
auch noch ausserordentlich scharfen Gegenkritik zu reagieren vermochte: „Verwies Schaffner auf Boccaccio, Goethe ... so hielt ihm Musil entgegen, der Schriftsteller lebe heute nicht von Goethe, Hebbel und Hölderlin, sondern von Mach, ... Einstein ... Russell und Peano, weil heute aller seelischer Wagemut in den Naturwissenschaften" zu finden sei. ${ }^{10}$ Solches Empfinden galt damals bis in den privatesten Bereich hinein, mit wundersamen Verästelungen bis hinein in den intim-erotischen Bereich. Während die Romantiker, E.T.A. Hoffmann allen voran, die Liebenden als „Leydener Flaschen“ darzustellen vermochten, zwischen denen der „electrische Funke“ übersprang, wie immer auch eine trennende Glaswand zwischen ihnen stand, wählte Musil vergleichbar mesmeristischen Metaphern sogar noch für das eigene Liebesleben. Seine Frau Martha hatte sich längst für ihn entscheiden. Der Mann begehrte aber weiterhin polygam die Schauspielerin Ida Roland. Denn: Diese sei ,geladen mit Nervosität, die aber nicht als Schwäche, sondern als Elektrizität wirkt."11 Die Elektrizitätsmetapher war also, und spätestens seit der Frühromantik, etabliert als das Bild überspringender Erotik; eine selbstverständliche Folge der damals angesagten neuen Zeit, und das noch bevor die Aufspaltung der wissenschaftlichen Welt in ,zwei Kulturen" erfolgt und festgeschrieben war. Und da sollte die Begegnung Kafkas mit Einsteins Theorien, die zudem als eine beweisbar intensive vor sich ging in ihrer Vermittlung durch Einsteins Lieblingsassistenten Ludwig Hopf, nicht dazu geführt haben, dass im Fall des Pragers eine neue Romanform entstand, analog zu Dürrenmatts Dramen? Zumal die angesprochene Revolution des Weltbildes durch die neue Physik so umfassend sich gestaltete, dass von Monokausalität hier schon, bedingt durch die Sache selbst, die Rede nicht sein kann. Zudem stand dagegen der Topos der „Ruinen“, den Franz Kafka wie dargestellt wählte, und der eben nicht mehr der feste Turm des Goethe'schen Bildungsromans gewesen ist, von dem aus sich die Bahnen der Auszubildenden hatten zuverlässig festlegen lassen (damit sie sich nicht zu Bohemiens entwickelten, sondern zu praktischen, soliden Medizinern, vorzüglich zu „Wundärzten“). Kafkas schon ganz frühe „Ruinen“-Empfinden dagegen verrät, dass hier einer ins Leben

\footnotetext{
${ }^{10}$ Karl Corino, a. a. O., S. 399.

${ }^{11}$ Zitiert nach Karl Corino, Robert Musil, a. a. O., S. 441. Kafka wurde übrigens von Musil gekannt und geschätzt, allerdings im Schatten der für Musil typischen Selbst-Verehrung, s. dazu Karl Corino, Robert Musil, a. a. O., S. 1552 insbesondere. Während der Zeit ihrer Bekanntschaft kann Musil die grossen Romane Kafkas noch nicht gelesen haben: „Es dürfte im Jahre 1923 oder 1924 gewesen sein, Kafka war jedenfalls noch am Leben, seine Romane waren noch nicht veröffentlicht.“ (Corino, Robert Musil, a. a. O., S. 1752).
} 
trat, dessen Schreiber-Existenz den klassischen Massstäben der von Heine so benannten „Kunstperiode“ gar nicht mehr entsprechen konnte. Franz Kafka, der „,kleine Ruinenbewohner“, der ohne den seine Existenz bestimmenden, 1914 ausbrechenden und das pränationalistische Europa vernichtenden „Grossen Krieg“ gar nicht zu denken ist, begann seine Tagebuchaufzeichnungen mit der Verzeichnung des „Halley’schen Kometen“. Der stand damals, 1911, Nacht für Nacht schweifbewehrt am Himmel über Prag. Prophezeite Krieg und Ordnungsauflösung. Die fand dann auch statt, gesellschaftlich-historisch sowie auch auf dem als so „objektiv“" geltenden Gebiet der Naturwissenschaften, und dort zuvörderst bei den „Physikern“, von wilhelminischen Geistern wie Dilthey bereits in den ersten Anfängen verängstigt registriert. Das Feld schien derart bereitet für die folgende gesellschaftlich-ästhetische Revolution, die gerade auch politisch, als der „Tod des Doppeladlers“, den altösterreichischen Beamten Kafka in Prag sehr wohl erreichte, womöglich noch intensiver, als hätte er in Wien gelebt.

\subsection{Vorläufer: Brockman und Shaffer samt Durs Grünbein}

Der gesamte Komplex wird später noch Gegenstand eines Diskurses sein, der sich zwischen Johann Wilhelm Ritters Physik als Kunst und dessen späterem Lebensbericht, zwischen Dürrenmatt und Brecht, und bis hin zu Durs Grünbeins und zu Enzensbergers so lesenswerten Beiträgen zu diesem Thema erstrecken muss. Hier geht es einleitungsweise erst einmal um die Vorläufer des eigenen Vorhabens. Sie sind überschaubar, liegen beide ca. zwei Jahrzehnte zurück, sind jeweils ausschliesslich in Englisch gehalten (wenn auch der eine mit deutschem Verlag, Erscheinungsort Berlin und New York). Den Anfang machte John Brockman, dessen The Third Culture 1995 im renommierten Simon \& Schuster Verlag in den USA erschien und schon ein Jahr später als dänische Ausgabe vorlag: Den Tredje Kultur. Hinsides den naturvidenskapelige Revolution. ${ }^{12}$ Brockman arbeitete als Literaturagent, und seine erste Absicht muss gewesen sein, seiner (nahezu durchgehend naturwissenschaftlichen) Klientel Zugang zu jenem Markt zu verschaffen, den er im Bereich der literarischen Kultur vermutet haben muss. Er wie auch sein dänischer Vorwortautor Tor Nørretranders unterstrichen, dass die Trennung der beiden seit C. P. Snow festgezurrten ,Zwei Kulturen“ verhängnisvoll, ungerecht

\footnotetext{
${ }^{12}$ Viborg 1996.
} 
(laut Brockman vor allem für die Naturwissenschaftler), - aber auch dabei sei, überwunden zu werden. Es sollte aufgeräumt werden mit dem Sachverhalt, dass der, der kein Wissen um die Einstein'sche Theorie, wohl aber über die Sonette Shakespeares besitzt, auf jeder europäischen oder amerikanischen Party gleichwohl als zureichend gebildeter, womöglich noch besonders „feinsinniger“ Gesprächspartner gelte. In der Frankfurter Allgemeinen Zeitung begrüsste damals Sybille Anderl auch deshalb den Brockman-Band wie folgt: „Der amerikanische Visionär John Brockman nahm dies 1995 zum Anlass, eine ,dritte Kultur` zu fordern, in der beide Kulturen in enger Kommunikation ihre jeweiligen Stärken zu neuem kreativen Potential vertiefen." 13 Gemessen daran, bleibt freilich noch viel zu tun; erweisen sich die als bereits erfolgt beschworenen Kulturveränderungen als eher noch zu vollziehende?

Doch gab Brockman den Ton vor für die folgende Ausgabe eines weiteren Buchs zur „Dritten Kultur“, das dann freilich von der literarischen Seite her sich des Themas annahm - und die sog. „Postmoderne“ einbezog, die für Brockman und seine Klientel noch keine Rolle gespielt hatte. Der Sammelband von Elinor S. Shaffer über die Third Culture erschien dann 1998 (wie verzeichnet in Berlin und New York, im de Gruyter Verlag). Er muss hier ausführlicher diskutiert werden, weil er für das eigene Vorhaben relevanter scheint. „C.P. Snow had argued that in advanced Western society we have lost even the pretence of a common culture." Die Repräsentanten der traditionellen literarischen Kultur auf der einen Seite, und die der naturwissenschaftlichen auf der anderen hätten quasi nichts mehr gemeinsam. Das sei ein Missstand. Bereits Snow habe in der zweiten Ausgabe seiner bekannt gewordenen Vorlesung aus dem Jahre 1963 sich davon beeindruckt gezeigt, und aus den Reaktionen der Öffentlichkeit gefolgert, dass eine „Dritte Kultur“ „was possible and indeed already on the horizon." 14 Snow habe sich überzeugt gezeigt, die angestrebte „Dritte Kultur“ würde kommen; habe sie aber aufgrund seiner „English Education“ nicht sehen können. Die amerikanische Autorin hat diese ihre Behauptung nicht weiter entfaltet, ebenfalls aber gemeint, „Social history“ könne die Lücke füllen. Denn Sozialhistoriker seien die, die beide Sprachen sprechen könnten, in einer Zeit, wo „moral sciences“ gefragt seien, die die Literatur als Gefäss gesellschaftlicher Veränderungen traktieren würden und so auch Texte wie Euklids Elemente, Newtons Principia und Darwins Origin oft the Species mit einschlössen. Das

\footnotetext{
${ }^{13}$ FAZ, 21.V. 2019.

${ }^{14}$ Elinor S. Shaffer, The Third Culture, Berlin, New York 1998, Introduction, S. 1.
} 
traf ins Zentrum. Nunmehr seien Mediatoren gefragt wie etwa George Levice, One Culture: Essays in Science and Literature aus dem Jahre 1987. Wiewohl Shaffer den Begriff des „New Historicism“ nicht erwähnt, erscheint unbestreitbar, dass dieser ihre Intentionen aufgenommen hat. Da er die eigene theoretische, eben sozialhistorische Fundierung beschreibt, sei er im Folgenden ausführlicher skizziert - nachdem noch Durs Grünbeins Position zur Darstellung gelangt ist, die unter manchen Aspekten einen Gegenentwurf zu Brockman wie Shaffer darstellt.

Grünbeins Theorie macht hier auch deshalb den Abschluss, weil sie am radikalsten ausgefallen ist, indem sie die beiden Kulturen nicht miteinander zu versöhnen, sondern die gesamte Diagnose von Snow zu „Schnee von gestern“ $\mathrm{zu}$ machen versucht, also ihr insgesamt die Basis zu entziehen trachtet. Das ging so: Wie bekannt, hatte der Physiker, Romanautor und hohe britische Staatsbeamte Charles Peirce Snow im Jahr 1959 einen Vortrag gehalten, der beachtliche Folgen haben sollte: The two cultures and a second look. Darin konstatierte der Brite eine hoffnungslose Entfremdung zwischen den beiden Kulturen, die sich nichts mehr zu sagen hätten und in gegenseitiger Ignoranz verharrten. Für Durs Grünbein ging es nicht mehr, wie noch für Brockman und Shaffer (und auch für den hier vorgelegten Text) um die Synthese in einer „Dritten Kultur“; sondern wesentlich um eine Widerlegung der Snow'schen Diagnose. Das rührte aus einem konkreten Anlass: Grünbein hatte 1995 den Georg-Büchner-Preis verleihen bekommen, und für ihn geriet Büchner $\mathrm{zu}$ der literaturgeschichtlichen Widerlegung der gesamten Lagebeschreibung bei Snow. Also Georg Büchner, der Autor revolutionär umstürzender Theaterstücke, - der damals 23-jährige Naturwissenschaftler hatte 1836 in seinem Zürcher Studierzimmer nicht nur die Schädelnerven seziert, sondern auch die Trennung der Literatur von der modernen Naturwissenschaft überwunden, indem er mit harter Grammatik und kaltem Ton in seinen Revolutionsstücken u. a. den verletzlichen menschlichen Körper beschrieben hatte. Der ,,ungeheure Riss in der Schöpfung“, den Büchner bekannter Massen auf diese Weise konstatierte, hatte sich ihm zum unheilbaren Weltriss zwischen Dichtung und Naturwissenschaft ausgeweitet. Der BüchnerPreis-Träger Durs Grünbein wiederum sah die zeitgenössische Variation dieser Thematik in den Romanen von Dietmar Dath, Daniel Kehlmann und Julie Zeh anwesend, und ferner in den Gedichten von Hans Magnus Enzensberger, Raoul Schrott und - eben seinen eigenen. Solche „,somatische Poesie“ besässe, das meinte der Preisträger, sogar ein konkretes Ursprungsdatum: Nämlich den Florenzer Vortrag des damals 24-jährigen Galileo Galilei über Dantes Göttliche Komödie. Denn darin hatte es der Astronom und Physiker unternommen, die Dante'sche Hölle mit physikalisch-geometrischen Mitteln zu vermessen. Sie 
sollte in einer Vollmondnacht des Heiligen Jahres 1300 stattgefunden haben; der Ort des Infernos hätte nahe an Neapel (und damit am Vesuv) gelegen. Seitdem, so analysierte Grünbein, wären die Wege der Naturwissenschaft und die der Künste energisch auseinander gegangen - und hätten einander nie wieder gekreuzt. Das Apodiktische wie auch das Aparte dieser These machen vorsichtig. Gewiss will sich ihr Urheber auf Quantenmechanik, Astronomie, Hirnphysiologie, Kybernetik und Ethnologie berufen, wobei seine Aufmerksamkeit in Büchners Nachfolge sich auf die Schädelnerven und insgesamt das Gehirn richtet. Mit Enzensbergers Gedicht Unter der Hirnschale findet der Preisträger ein unaufhörliches Ticken und „Feuern“ und unablässiges gegenseitiges Beobachten in dieser „neuronalen Metropole" vor allem bei Nacht vor, doch es kommt bei ihm zu keinerlei Synthese des Gegensätzlichen; sie wird, anders als bei Shaffer und Brockman, auch gar nicht erst angestrebt. Unbeschadet der bewunderswert hohen lyrischen Dichte, die die Verse Durs Grünbeins als die eines „,poeta empiricus“ aufweisen, bleibt die theoretische Schlussfolgerung eher allgemein: „Was die Dichtung mit der Naturwissenschaft verbindet, ist die Fülle der Einzelbeobachtungen, Spezialdiagnosen, Schock- und Glückserlebnisse, die man machen muss, um das, was am Menschen menschlich ist, zu verstehen. Zwar scheint nicht unbedingt die Literatur dazu berufen, die Zukunft der Naturwissenschaft zu beeinflussen. Aber die Gegenwartslyriker können Snows These von der halbierten Kultur durchaus als ,Snow von gestern" widerlegen." 15 Letzteres ein gelungenes Wortspiel; ob es mehr ist als ein solches, kann nur methodologisch entschieden werden, in intensiver Auseinandersetzung beispielsweise mit dem "New Historicism“ Stephen Greenblatts. Dem wenden wir uns nun zu.

\section{2 „New Historicism"}

Es gilt dabei, ein wenig auszuholen. Während ein substantieller Teil der KafkaForschung in den siebziger und achtziger Jahren des vergangenen Jahrhunderts Kafkas Texte erdnah und tiefschürfend auf ihre biographische Verbindlichkeit hin las, und mancher beispielsweise Milenas Mann Ernst Polak im Klamm des Schloss' wiederzuerkennen meinte, turnte unter der Zirkuskuppel der Erzähltheorie bereits ein anderer Teil des Gewerbes, der Kafkas Texte als Grenzexperimente des Erzählens überhaupt verstand (und dabei mehr und mehr in

\footnotetext{
${ }^{15}$ Michael Braun, Die deutsche Gegenwartsliteratur, UTB Köln, Weimar, Wien (Böhlau Verlag) 2010, S. 174.
} 
den postmodernen Luftraum geriet). Während auf der einen Seite Kafkas TextLogik zugunsten biografischer Rekonstruktion in Gefahr geriet, dispensiert zu werden, verschlang auf der anderen Seite der Text den Autor. Zugespitzt formuliert: Hie erdnahe biografistische Verdinglichung; dort poetologische Trapeznummern. Zwischen Skylla und Charybdis einen Weg zu finden, das verlangt, im Bilde zu bleiben, geradezu des Odysseus' Wegfindekunst und eine genaue, nüchterne Sicht auf die Wegmarken, wie vor allem der Text sie setzt (aber ohne dabei sich auf Textimmanenz zu beschränken). „Phronesis“ hieß diese Geisteshaltung vor Zeiten. Odysseus kannte das Wort, noch Elias Canettis Eigene Lebensbeschreibung so viele Jahrhunderte später folgte ihm. Woraus folgt: Um an der Insel anzulanden, die Franz Kafka tatsächlich bewohnte, müssen wir deren Koordinaten vermessen. Sie aber ergeben sich vor allem aus den Diskursen der Zeitgenossen, und, das macht hier den Unterschied, vor allem aus denen der naturwissenschaftlich gebildeten und interessierten Zeitgenossen à la Robert Musil. Im Idealfall also aus den Überlegungen derer, die neue, umstürzende aber doch auch belegbare Theorien, tendenziell solche mit der Sprengkraft der Einstein'schen, entwickeln. Auf dieser Karte, auf der die „sozialen Energien“ (ein Terminus, geprägt von Stephan Greenblatt im Rahmen des „New Historicism“) mit allen ihren Untiefen, Klippen und Strömungen verzeichnet sein sollten, ist erst der Platz zu verorten, den Kafka „tatsächlich“ innehatte: - so sagt jener philosophische common sense als englischer Nachfolger der beschworenen „Phronesis“. Über jenen Franz Kafka, der belegbar und jenseits allen Zweifels ein Gesprächspartner Albert Einsteins und darüber hinaus Zuhörer von Vorträgen von Einsteins „Privatassistenten“ Ludwig Hopf über das neue Weltbild gewesen ist, wie es sich damals aus der Relativitätstheorie ergab. Jener Kafka auch, der selbstverständlich und auch bewusst ein Sohn der Moldau-Metropole gewesen ist, in der von Keplers Sphärenharmonie bis hin zum Meyring'schen Golem (und dann eben auch Einsteins Theorien) die Suche nach den inneren Bewegungsgesetzen der Welt seit Jahrhunderten ihren angestammten und bevorzugten Platz gehabt hatte. Vor diesem - zugegebenermaßen pointiert dargestellten - Hintergrund nun also der Versuch, „mit den Toten ins Gespräch zu gelangen“. Wie aber stellt man das an? Wie gesagt: Die damaligen Diskurse der deutsch sprechenden böhmischen Judenheit wären in ihren prägenden Zügen in Prag zu rekonstruieren, und die würden eben auch Einstein samt „Privatassistent“ Hopf aus Süddeutschland einschliessen, und somit die Hoffnung des späten Snow auf Überwindung der Zwei-Kulturen-Kluft realisieren? So könnte es vielleicht gelingen, die Kraftlinien in jenem Feld sozialer Energie zu markieren, innerhalb dessen Kafka sich im Hochsommer 1911/1912, also noch vor Ausbruch des Ersten Weltkriegs, bei 
seiner ersten Begegnung mit Einstein geistig bewegte. Gewiss: Was die bereits mehrfach bemühte soziale Energie angeht, so kann man nur, übrigens zusammen mit Greenblatt selbst, konzedieren, dass diese keinerlei messbare Größe darstellt. Sie lässt für monokausale Ableitungen keinen Platz. Wird aber dennoch in ihren Auswirkungen im jeweiligen Kunstwerk registrier- und ablesbar. Denn nicht nur von Shakespeares Königsdramen, auch von den drei Romanen des Pragers gilt, was Greenblatt unter dem Stichwort der sozialen Energie aufgeschrieben hat: „Während die meisten kollektiven Äusserungen, die aus ihrer ursprünglichen Umgebung herausgerissen und an einen neuen Ort oder in eine neue Zeit transportiert werden, nur noch als Leichen ankommen, gelingt es manchen von ihnen, kraft der in ihnen codierten sozialen Energie, über Jahrhunderte hinweg die Illusion der Lebendigkeit wachzurufen. Mir geht es darum, die Verhandlungen zu verstehen, kraft derer die Kunstwerke eine solche wirkungsvolle Energie erhalten und verstärken.“16 Immer geht es dabei um „Energie“, in der Physik ebenso, wie in der Philosophie und in der von Greeblatt inspirierten Philologie.Der mit dem Weltkriegsausbruch entstandene Process wurde beispielsweise in Norwegen im Jahre 2000 zum „Roman des Jahrhunderts“ erklärt. Kaum jemand auf der gesamten Erde wird dieser Wahl widersprechen wollen. Doch warum? Was für eine soziale Energie hat sich in diesem Buch erhalten? Zwar, der New Historicism bezieht sich laut Selbstaussage keineswegs auf die deutsche „Historische Schule“, den „Historismus“ etwa eines Johann Gustav Droysen. In der Bezeichnung sollte vielmehr ein konkurrierender Bezug zum „New Criticism“ laut werden - eine Absetzung, die dennoch das Prinzip des ,close reading“ beibehielt, allerdings nur, um es um die, ganz unverzichtbare, (kultur)historische Dimension der Texte zu ergänzen. Wie sehr sich beides als Verfahren gerade gegenüber ,tot interpretierten" Autoren, wie beispielsweise Shakespeare oder Kafka, eignen kann, liegt auf der Hand. Hier wiederum hat der ebenso spielerische wie elegante Chiasmus von der "Geschichtlichkeit der Texte und der Textualität der Geschichte“ seinen Platz, den Louis Montrose geprägt hat. Nur: „Neu“ ist auch dies nicht. Der grosse Systemphilosoph Hegel hat vor rund 200 Jahren in seiner Philosophie der Geschichte geschrieben: „Die Geschichte verbindet in unserer Sprache sowohl die objektive als auch die subjektive Seite. Sie meint die res gestae (das Geschehene) und die historia rerum gestarum (die Erzählung des Geschehenen)." ${ }^{17}$ Aussagen eines sogenannten ,toten weissen Mannes“? Zitieren wir also Montrose, der noch

\footnotetext{
${ }^{16}$ Stephen Greenblatt: Verhandlungen mit Shakespeare, Berlin 1990, S. 12.

${ }^{17}$ Zitiert und diskutiert bei Amos Funkenstein: Jüdische Geschichte und ihre Bedeutung. Frankfurt/Main 1995, S. 11.
} 
am Leben ist: „Mit der Geschichtlichkeit von Texten behaupte ich die These von der kulturellen Bestimmtheit, der gesellschaftlichen Einbettung jeglicher Art von Geschriebenem ... Mit der Textualität von Geschichte behaupte ich die These, dass wir erstens keinen Zugang zu einer vollen und authentischen Vergangenheit haben, zu einer gelebten materialen Existenz, die nicht über die überlebenden textuellen Spuren der betreffenden Gesellschaft vermittelt wäre..."18 Keinen Zugang zwar zur ,vollen und authentischen Vergangenheit"; aber doch zu einer abgeschwächt authentischen, gleichsam eingeschränkt rekonstruierbaren? Statt der illusorischen Rekonstruktion des „Wie es eigentlich gewesen“ eben offene „Verhandlungen“ mit den Toten und ihrer Epoche. Wobei ,Verhandlungen“ immer nur meinen kann, die historische Distanz zwischen Gegenwart und Vergangenheit eben nicht naiv einzuziehen. Sondern, im möglichst genauen Hinhören auf die zeitgenössischen Diskurse, die soziale Energie zu bestimmen und zu vermessen, aus der heraus jedes große Kunstwerk entstand, und die es auf die ihm je eigene Weise transportiert als eine gleichermaßen historisch bestimmte und dennoch überzeitliche, im Glücksfall also unvermindert gültige Größe. Das Verstehen der Stimmen der Toten also entmystifiziert zur Rekonstruktion der Epochen-Diskurse in ihrer ganzen Vielstimmigkeit und in möglichst unverkürzter Breite. Wobei nicht eigens unterstrichen werden muss, dass der Terminus „Verhandlungen“ ganz grundsätzlich (und darin notwendigerweise anti-postmodernistisch) auf den dialogischen Charakter von Literatur verweist, und mithin auf das hermeneutische Grundmodell des Gesprächs. $\mathrm{Zu}$ erkennen wäre ferner, dass solche ,Verhandlungen" in einer Tradition von Platon bis Heidegger, von Sokrates bis Gadamer stehen. Hier scheint also eine grundsätzliche Korrektur am Platz, weil unser folgender Text auf jener interdisziplinären und grundlegenden Plattform beruht, die kein anderer als Werner Heisenberg in Philosophie und Physik (bereits vor Erscheinen der hier vorgestellten Sammelwerke zu einer „Dritten Kultur“ und lange vor der Dominanz der „Postmoderne“) vorgelegt hat. Was neu ist, ist aktuell - aber nicht notwendigerweise auch ,wahr" und somit von längerer Verweildauer. Ich möchte in diesem Zusammenhang eine Passage aus Johann Gustav Droysens Historistik von 1868 zitieren. Also aus einem rund 150 Jahre alten Text, in dem ich nicht nur eine verblüffende Nähe zum New Historicism, beispielsweise in der Metaphernwahl, entdecke, sondern, horribile dictu, auch noch mein eigenes „Erkenntnisinteresse“ beschrieben finde. Ein notwendig längeres Zitat: „Unsere

\footnotetext{
${ }^{18}$ Louis A. Montrose: Die Renaissance behaupten. In: New Historicism, hrsg. v. Moritz Basler. Frankfurt/Main 1995, S. 67.
} 
Aufgabe kann nur darin bestehen, dass wir die Erinnerungen und Überlieferungen, die Überreste und Monumente einer Vergangenheit so verstehen, wie der Hörende den Sprechenden versteht, dass wir aus jenen uns noch vorliegenden Materialien forschend zu erkennen suchen, was die Formenden, Handelnden, Arbeitenden wollten, was ihr Ich bewegte, das sie in solchen Ausdrücken und Abdrücken ihres Seins aussprechen wollten. Aus den wie immer lückenhaften Materialien suchen wir sie, ihr Wollen und ihr Tun, die Bedingungen ihres Wollens und die Wirkungen ihres Handelns zu erkennen; wir suchen aus den einzelnen Äußerungen und Formgebungen, die wir noch fassen können, uns ihr Ich ... zu rekonstruieren und aus der so gewonnenen Erkenntnis die zerbröckelte und verwischte Perspektive ihres Gesamt-Daseins zu ergänzen ..."19 Denn: „Im Deutschland der Gründerzeit hatte dieser Historismus eine besondere Färbung angenommen. Der Historismus blickte in die Geschichte zurück, um sich ins Bewusstsein zu holen, wie herrlich weit man es doch gebracht habe. Zugleich aber galt es, eine Unsicherheit im Lebensgefühl und im Stil zu kompensieren. Man wusste noch nicht so genau, wer man war und worauf man hinauswollte. Und so verband sich dieser Historismus auch mit der Lust an Nachgemachten, am Unechten."20 Eben letzteres verweigert und überwindet Greenblatts „New Historicism“. Deshalb kann er sich der "Neue“ nennen (und mit zahlreichen Preisen ausgezeichnet werden, in Norwegen mit dem prestigeschweren HolbergPreis). Franz Kafkas „Ich“, dessen Konzentration im inzwischen weltweit aufgerufenen „Kafkaesken“, und darüber hinaus das „Gesamt-Dasein“ des Prager Autors aus seiner Epoche heraus eben auch im Sinne Droysens zu ,verstehen“: Darauf zielt dieser Text ab. Nichts anderes meinte, übrigens, bereits Goethes schlichter Vers „Wer den Dichter will verstehen/ Muss in Dichters Lande gehen“. Nichts anderes meinten auch die beherzigenswerten Sätze, die der Prager Willy Haas, seinerseits mit Kafka sehr gut bekannt und erster Herausgeber von dessen Briefe an Milena, in Erinnerung an seine „Jugendtage“ im Jahr 1957 formuliert hat: „Franz Kafka, und er allein, hat die Welt unserer Jugend chiffriert und in ein

\footnotetext{
${ }^{19}$ Johann Gustav Droysen: Historistik. München 1967, S. 26 f. Für den Hinweis auf das Zitat danke ich Rainer Benjamin Hoppe, und will an dieser Stelle auf dessen Dissertation Horizont aus Schlagbäumen ...? verweisen (Bd. I des Jahrbuchs Polygon des „Institutt for moderne fremmedspråk“ der NTNU/Trondheim, Aachen 2006; Vorläufer von „Electrischer" Prometheus, als dessen erster Band dieses Buch nun erscheint. Die Darstellung des „New Historicism“ wurde von mir, mehr oder weniger variiert, auch in anderen Arbeiten präsentiert)

${ }^{20}$ R. Safranski, Romantik, a. a. O., S. 431.
} 
paar grossen, monumentalen, wenngleich fragmentarischen Gemälden zusammengefaßt, vor allem im Process und im Schloss ... Kafka hat alles gesagt, was wir zu sagen hatten und nicht gesagt haben, nicht sagen konnten. Dies ist für mich sein Genie. Ich kann seine Bücher wie im Traum lesen. "21 Lesen wir diese Bücher also neu, unsererseits inspiriert vom Vorhaben eines „dritten“, synthetisierenden und transdisziplinären Verstehens, das sich zudem aus einem Zusammenfallen des ältesten romantischen mit dem immer noch neuen und uneingelösten, utopisch-freudianischen Paradigma speist: „Es rührt sich, schreibt (Herbert) Marcuse, die erotische Energie der Natur - eine Energie, die befreit werden will: auch die Natur wartet auf Revolution. Das hätte auch Novalis sagen können. "22 Nur dass bei Novalis eine Gedanken- und Gefühlsrevolution intendiert war; während der Jüngere meinte, die reale Gesellschaft revolutionieren zu können - das alles freilich nicht, ohne einige höchst lesenswerte Bücher zu hinterlassen.

\subsection{Fragen der Vereinbarkeit von Postmoderne und "Dritter Kultur"}

Ohne Frage zielt auch Elinor S. Shaffers Einleitung, um auf sie zurückzukommen, auf eine solche neue Qualität des (hermeneutischen) Verstehens. Nicht nur die Frage nach dem Entstehen des Universums sei neu zu beantworten auf dem Hintergrund der fortgeschrittenen Physik des 21. Jahrhunderts, schreibt sie. Die Molekularbiologie komme hinzu, etwa Watson mit The Double Helix, ferner die Chemie und die Genetik, beide wichtig für das Verstehen etwa des französischen Weltautors Michel Houellebecq. Solche Einbeziehung der einzelnen Naturwissenschaften aber verlange nach einer Rückbesinnung auf die (frühe) Romantik, und all dies wiederum, um einen allzu tumben, dogmatisch positivistischen Glauben an „harte Facts“ zu relativieren. Ritter, Novalis, Arnim, Schelling, Goethe, Coleridge, Shelley müssten in diesem Zusammenhang genannt werden. Denn: ,The need to find a framework in which old values could still guide the new procedures seemed urgent to many“. Eine Feststellung, die in

\footnotetext{
${ }^{21}$ Willy Haas: Aus Jugendtagen mit Werfel, Kafka, Brod und Hofmannsthal. In: Als Kafka mir entgegenkam, hrsg. v. H.- G. Koch, Berlin 1995, S. 78.

${ }^{22}$ Bei R. Safranski, Romantik, a. a. O., S. 597.
} 
unserem Zusammenhang zu unterstreichen bleibt. Danach gelangt die Herausgeberin zum Zeitgeist, den sie bereits ,moderner“ auffasst, als noch Brockman es tat: „That despite their differences Romanticism and Post-Modernism may have the similiar function of providing a half-way-house in which both arts and sciences may be accommodated.“23 Zwar, die „interdisziplinary interests and contributions" müssten sich auch und gerade auf Ökologisches beziehen; immer müsse gefragt werden: „Has Nature a Future“. Und bei dieser Art neuer Hermeneutik kämen dann auch Chaos-Theorie und Heisenbergs „Unschärferelation" notwendig ins Spiel. ${ }^{24}$ In der Nachfolge von Jeremy Adlers The Aesthetics of Magnetism, wo Goethe und Schelling neben Novalis und Ritter, Mesmer und Schlegel behandelt würden, müsse man sich an den Arbeiten von Calvino, Barthes und Thomas Pynchton orientieren, denn die neu auftauchenden Strukturen glichen doch tatsächlich denen um $1800 .{ }^{25}$ Zwar wolle man kein neues Lehrgedicht nach dem Muster des Lukrez schreiben, aber doch entschieden festhalten: „The approach of the humanities to the sciences is in our time not just curiosity or a diversion ... but a central feature of intellectual life. "26 Es ist äusserst bedenkenswert, dass Hans Magnus Enzensbergers Essay zu diesem Thema, später in unserem Text noch eingehend behandelt, zu recht identischen Konklusionen (Calvino, Pynchton, Lehrgedicht) gelangt. Vielleicht hatte Brockman doch Recht mit seiner Annahme, die Zeit einer „Dritten Kultur“ sei bereits angebrochen und wirksam?

Und doch ist damit ein Wesentliches übersehen: Nämlich, dass die Wissenschaftsbesessenheit der Frühromantiker, wie immer unscharf zu nennen gemessen am Standard der Moderne (eben weil diese ihrerseits durch den Positivismus mitbegründet wurde), bereits damals dem zentralen „Anything goes“-Postulat der Postmoderne so diametral wie fundamental entgegenstand. Um diese Frage geht es; ,hier liegt der Hund begraben“, wie der grosse Stilist und Sarkastiker Georg Friedrich Wilhelm Hegel zuweilen sich auszudrücken pflegte. Denn vermag sich auf die - immer noch exakten, daran ändert Heisenbergs „Unschärferelation" nicht das Geringste - Naturwissenschaften jemand zu berufen, der Beliebigkeit nicht nur verzeiht, sondern sogar zum unterhaltsamen Ideal erhebt? $\mathrm{Zu}$ dieser Frage ist neuerdings entscheidendes Material zu finden bei Laurent

\footnotetext{
${ }^{23}$ E. S. Shaffer, The Third Culture, a. a. O., S. 11/12.

${ }^{24}$ E. S. Shaffer, The Third Culture, a. a. O., S. 7.

${ }^{25}$ E.S. Shaffer, The Third Culture, a. a. O., S. 8 und 9. Hier auch eine differenzierte Begründung der zitierten Aussage.

${ }^{26}$ E. S. Shaffer, The Third Culture, a. a. O., S. 12.
} 
Binet, Die siebente Sprachfunktion. ${ }^{27}$ Die Antwort kann nach erfolgter Lektüre nur ein striktes Nein seien. Und sie wird illustriert, eine ironisch-satirische Volte der philologischen Vernunft, ausgerechnet dadurch, dass der führende Postmoderne Jaques Derrida, gerade da, wo er Kafka verstehen wollte, dieses „Nein“ unabweisbar machte - allerdings auf amüsante Weise. Davon noch später. Der exemplarische Postmoderne hatte die Wahrheit übersehen, die am Beispiel des Odysseus sowohl Adorno/Horkheimer in Dialektik der Aufklärung, sowie später Rüdiger Safranski gewonnen haben: Dass bereits Nietzsche erkannt hatte, dass man einerseits mit dem Leben Fühlung behalten müsse, ohne aber die zivilisatorischen Schutzvorrichtungen, zu denen eben auch die logische Argumentation gehört, aufzugeben um des sophistischen Spasses willen. Gerade Odysseus verkörpert darin eher apollinische, wie es auch Safranski erkannt hat, denn dionysische Weisheit. Der homerische Held will nicht auf die ungeheure, weil potenziell existenzvernichtende Erfahrung der verlockend singenden Sirenen verzichten. Daher akzeptiert er seine eigene Fesselung durch die stabilisierenden Gewohnheiten der Kultur; gestattet seiner Mannschaft, ihn an den Mast zu binden, während die nackten Sirenen ihn ins Verderben hinunterzuziehen trachten, sich dabei geradezu schmerzvoll verrenkend. Insofern hat auch Franz Kafka Recht, der diesen Mythos ebenfalls mit einem kurzen Text bedacht hat, in dem die Sirenen aber „schweigen“, - offenbar zum Schweigen gebracht durch die notwendige, aber stabilisierende Langeweile der europäischen Kultur mit ihrer zentralen Forderung nach Selbstbeherrschung? Was freilich der Odysseus des Pragers nun auch wieder nicht ertrug. Gerade das Schweigen der Fabelfrauen wurde ihm unerträglich. Denn Franz Kafka gehörte, wie immer ein altösterreichischer Beamter, dennoch in seinem Wesenskern zu jener neuen Romantik, die im Wien der Jahrhundertwende sich beispielsweise mit dem Namen Hugo von Hofmannsthal verband. Dessen Schicksal stand dem des Pragers nicht so fern: Von den jungen revolutionären, lyrischsubjektiv gestimmten Anfängen (bei Kafka in Beschreibung eines Kampfes) hin zur qua Literatur betriebenen, wertschweren Konservativen Revolution. Denn auch Kafka imaginierte sich am Schluss als eine der wenigen noch tragenden Säulen Altösterreichs, was wenig bekannt, dennoch unabweisbar erscheint. ${ }^{28}$

\footnotetext{
${ }^{27}$ Hamburg 2017. Das Buch erscheint als eine ebenso schonungslose wie respektvolle Abrechnung mit Jaques Derridas zeitweiliger Alleinherrschaft über die Humaniora.

${ }^{28}$ Kafkas Prager Gesprächspartner Gustav Janouch hat Ähnliches erinnert und wurde dafür scharf attackiert in den Zeiten des postmodernen Begriffszaubers, von Enzensberger dann das „Kasperletheater der Dekonstruktion“ geheissen. Dazu ausführlicher auch Bernd Neumann, Der andere Franz Kafka, Würzburg 2018, S. 15 ff.
} 
Freilich teilte Kafka nicht den mittelterlich-philosophischen Nominalismus, den Hofmannsthal bei seinem Lehrer Ernst Mach kennengelernt hatte; wohl aber Hofmannsthals politisch-konservative Instinkte, und ebenso die imperative Erkenntnis, dass in der modernen Literatur „das Atom zu spalten“, und „mit den Sternen Ball zu spielen“ sei. Davon später noch mehr. Zudem ist beispielsweise in der zentralen dionysischen Messer-Phantasie der Kafka'schen Beschreibung eine Kampfes (Max Brod wird in seinem Tycho Brahe darauf antworten) durchaus eine Nähe zur damals offenbar alltäglichen dionysischen Feststimmung unter den führenden Intellektuellen deutlich: „Da trat Stefan George als Caesar auf, Schuler als Urmutter Gaia, Wolfskehl als Dionysos. Man tanzte im Bacchus-Reigen, blies die Panflöte, lagerte auf Tigerfellen und entzündete blau schimmernde Ampeln. Nymphen aus dem Münchner Randbezirken wurden geladen, auch stämmige Bauernburschen, die zur Not den heidnisch-germanischen Urgeist verkörpern konnten." ${ }^{29}$ Prag lag damals nicht allzu weit von München entfernt. Kafka hatte in der Isar-Metropole seinerseits gelesen und mit seinen sadistischen WollustPhantasien in der Strafkolonie einigen Damen ihre charmant-zeitgemässe Ohnmacht beschert. Von französisch-perverser Lüsternheit war dies bestimmt gewesen, wie sie damals europaweit aktuell erschien (und bei Brod wie bei Kafka als Monatsabonnement pornographischer Darstellungen handfest zugegen war), und auf diese etwas perverse (= ,umgekehrte“) Weise des Pragers eigenes Diktum auf besondere Art erfüllte: Wonach jedes gute Buch „die Axt für das gefrorene Meer" in uns sein sollte?

Jedenfalls: „Leben“ hatte damals, wie schon zuvor in der Frühromantik, einen neuen Klang gewonnen, geheimnisvoll, verführerisch und gegen die allgemeine, nur Solides wollende, „bloss lebendige Lebenszappelei“ (Safranski) der Philister nicht nur des ausgehenden 19. Jahrhunderts gerichtet. „Leben“ wurde zu einem neuen Zentralbegriff dieser untergehenden Welt, agitierend allemal, freilich unschärfer als Begriffe wie „Sein“ oder „Natur“, wie „Gott“ oder „Ich“ in den Epochen zuvor. Der die Beschreibung einen Kampfes niederschreibende Kafka ist denn auch nicht nur als eine Art Ernst Jünger der jüdischen Assimilation zu verstehen. Sondern ebenso als einer, den, darin eben doch wie Ernst Jünger, die damalige ,totale Mobilmachung ...,Verwandlung des Lebens in Energie, wie sie sich in Wirtschaft, Technik und Verkehr im Schwirren der Räder oder auf dem

\footnotetext{
${ }^{29}$ R. Safranski, Romantik, a. a. O., S. 472.
} 
Schlachtfeld als Feuer und Bewegung offenbart ${ }^{\star 30}$ affiziert und fasziniert hatte. Schliesslich schrieb Franz Kafka am Ende seines Lebens als ein Bewohner der fiebernden, vergnügungstollen Metropole Berlin, wo er sogar noch den zu Weltruhm und allgemeiner Anerkennung gelangten Albert Einstein besucht haben soll, der nun zentral in Berlin, dem damaligen Weltzentrum der modernen Physik, residierte; und trotzdem seine Zürcher Staatsbürgerschaft zu behalten wünschte, bereit zu jeder tollkühner Schwindelei in dieser Angelegenheit. Zum anderen richtete sich der angesprochene neue, ach- -so-vive Lebensbegriff noch immer gegen den seelenlosen Mechanismus des ausgehenden 19. Jahrhunderts, wollte er doch eine neue Einheit von Seele und Leib, und damit Dynamik und Kreativität stiften. „Es wiederholte sich der Protest von Sturm und Drang und Romantik. Damals war ,Natur' beziehungsweise ,Geist" die Kampfparole gegen Rationalismus und Materialismus gewesen. Der Begriff ,Leben“ hat jetzt dieselbe Funktion.“31 Zum „Lebens“-Begriff zählte damals auch der des existentialistisch entscheidenden „Augenblicks“. Er gehört als eine zentrale Kategorie zu der von Søren Kierkegaard entwickelten existentialistischen Theologie, war Kafka durch seinen dänischen „Blutsbruder“ nahe, mochte auch eine Verbindung in Form der beiden gemeinsamen Aufmerksamkeit auf Mozart und dessen Opern gebildet haben, die Witwe des „göttlichen Kindes“ lebte ja damals bereits in Kierkegaards Kopenhagen. Was damals bei Jünger „,plötzlicher Schrecken“, bei Tillich „Kairos“ und bei Carl Schmitt „Augenblick der Entscheidung“ hiess, hatte seine Karriere bei Kierkegaard begonnen; und war mithin auch dem Prager bekannt. Der Existentialphilosoph kam damals überhaupt in Mode. Bei ihm stand jener Augenblick im Mittelpunkt, in dem Gott ins Leben des Einzelnen einbricht, den Einzelnen zur Entscheidung auffordernd, den Sprung in den (absurden) Glauben zu wagen. Was bei ihm Einbruch hiess, war von Friedrich Nietzsche eher als Ausbruch gefasst worden, als Ausbruch aus dem allzu träge Gewohnten. Nietzsche, in Jugendzeiten noch der Komponist fromm-pietistischer Töne, konnte bei solchen Gelegenheiten erneut hymnisch werden: „Die grosse Loslösung kommt ... plötzlich, wie ein Erdstoss: die junge Seele wird mit einem Male erschüttert, losgerissen, herausgerissen ... ein aufrührerisches, willkürliches, vulkanisch stossendes Verlangen nach Wanderschaft.“"32 Zwar, mit

\footnotetext{
${ }^{30}$ Zitiert nach R. Safranski, Romantik, a. a. O., S. 520.

${ }^{31}$ R. Safranski, Romantik, a. a. O. S. 461 f.

${ }^{32}$ Zitiert nach R. Safranski, Romantik, a. a. O., S. 524. (In diesem Fall macht Safranskis essayistische Zitierweise eine Identifizierung des Zitats bei Nietzsche unmöglich).
} 
Kafkas Assimilations-Wanderschaften im Schloss und anderswo hatte dies nicht unmittelbar zu tun. Dennoch sind es auch dort immer die (verpassten) „Augenblicke", die die besondere Aufmerksamkeit des Erzählers erhalten. Rüdiger Safranski hat deshalb in seinem Romantik-Buch die Brücke zu Kafkas Schloss mit seiner schläfrigen Beamtenschaft schlagen wollen. „Die Mobilitätsphantasien der Neuen Sachlichkeit werden von der Obsession beherrscht, man könne seine Zeit verfehlen, wie man einen Zug verpasst ... Von dieser Geistesgegenwart handelt auch Kafkas Roman ,Das Schloss', den man als zeitgemäss empfindet. Darin wird die verpasste Gelegenheit und mangelnde Geistesgegenwart zu einem metaphysischen Horrorszenario: Der Landvermesser Josef K. verschläft ein Stelldichein bei der Schlossbehörde. Vielleicht hätte es ihn retten können." ${ }^{33}$ Wohl eher nicht; denn K. stirbt an Erschöpfung, als er erfährt, dass er „gerettet“, also zur Assimilation angenommen ist.

Verpasste Chancen aber gibt es auch anderswo. Etwa ganz entschieden in Jaques Derridas Kafka-Deutung. Sie ist alles Mögliche, freilich nie von der Lebendigkeit der oben geschilderten lebensphilosophischen Aufbruchseuphorie überstrahlt gewesen, wie immer sie darauf wohl gehofft hatte. Mit Blick darauf ist festzuhalten: Es wird heute in der konturschärfenden Beleuchtung des schräg einfallenden Abendlichts erst wirklich erkennbar, welche Wirklichkeitsverluste aus dem letzten halben Jahrhundert postmoderner Philologie zu vermelden sind. Die folgenden Beispiele können den von Elinor S. Shaffer beschriebenen Zustand im immer noch heutigen "half-way-house“ schlaglichtartig beleuchten. Als eine unverzichtbare Standortbestimmung für den, der sich nach dem Ende der ,theory“-Dominanz, womöglich grade dadurch motiviert, mit dem so viel interpretierten Autor aus Prag neu befassen möchte. Die Postmoderne erreichte gerade den Zustand ihres ultimativen Höhenrauschs, als Jacques Derrida im Jahre 1982 (und das immerhin vor der „Royal Philosophical Society“ in London, hier hatte einst der grosse Newton vorgetragen; Derridas auf Englisch gehaltener Vortrag markierte dann auch des Franzosen Durchbruch zur Weltgeltung) in langanhaltender Rede über Kafkas Vor dem Gesetz sprach. ${ }^{34}$ Dass gerade die Postmoderne ausgerechnet an Kafka ihre (wie sie meinte: luftig-lockere) Lieblingsspeise besaß, wird nicht zufällig an deren Hauptphilosophen deutlich.

\footnotetext{
${ }^{33}$ R. Safranski, Romantik, a. a. O., S. 522.

${ }^{34}$ Gedruckt als „Devant la loi“, in: Kafka and the Contemporary Critical Performance: Centenary Readings. Hrsg. v. Alan Udoff. Bloomington 1987, S. 181 ff. Der Wichtigkeit von Derridas Vortrag angemessen, ist dieser auch in anderen meiner Arbeiten, mehr oder weniger variiert, dargestellt.
} 
Kafkas zentraler, im Roman Der Process enthaltener, wieder und wieder interpretierter Text diente dem Postmodernen als bloßes Spielmaterial, an dem er die eigene allumfassende Informiertheit funkeln ließ. Wie es damals im Schwange war, wollte der Philosoph gleich drei obsolet gewordene Paradigmen erschüttern (,to undermine the conditions of such a consensus"): Dass Kafkas Text eine eigene Identität beanspruchen könne; dass der Autor als eigene Instanz eine Rolle spielen dürfe; und dass es jenseits individuell-arbiträren Urteilens Gründe gäbe, Vor dem Gesetz und mithin den Process insgesamt als literarischen Text zu deklarieren. Im Rahmen der angesagten „neuen Unübersichtlichkeit“ bot Derrida folgende Lesarten an: Kafkas Text, da in ihm die Haare in des Türstehers Nase und in dessen Pelz eine wichtige Rolle spielten, könne auf die bei Freud angenommene Genese der Moral verweisen, besitze dabei doch der aufrechte Gang und die sich daraus ergebende erhöhte Position der Nase samt der solcherart erzielten Entfremdung des Menschen vom Olfaktorischen seine Bedeutung. Das sei eine Möglichkeit. Aber es könne sich auch um das (wie man weiß: In der Regel verborgene) „Sein“ Heideggers handeln, denn die Begriffe „Türhüter“ und der Heidegger'sche „Hüter des Seins“ verwiesen aufeinander. Dass „Hüter“ im Falle Kafkas die Konnotation des Ausschließenden, bei Heidegger aber die konträre des Beschützenden besitzt, musste den polyglotten Denker nicht weiter bekümmern. Zumal dann die dritte Derrida'sche Lesart erneut „the Freudian Turn" nahm und herausgefunden zu haben meinte, dass es sich bei Kafkas Text um die Thematisierung des coitus interruptus bzw. der ejaculatio praecox drehen müsse, möglicherweise um beides zugleich, ein Hermeneut oder gar Sexualtherapeut, wer hier genauer unterscheiden wollte. In jedem Fall aber, es gab ja damals bereits den Feminismus, sollte es um die Verweigerung der „Penetration“ gehen. Was wiederum, so die schlussendliche Aufgipfelung Derrida'schen KafkaVerständnisses, völlig damit übereinstimme, dass Kafkas Vor dem Gesetz die Undeutbarkeit aller Texte thematisiere: Man steht davor und kommt nicht rein. Festzuhalten als unhintergehbares Faktum ist dagegen, dass in Kafkas Text die Haare in des Türstehers Nase überhaupt nicht existieren. Derrida erfindet sie „dekonstruktivistisch“, um endlich zu seiner wissenschaftlich-olfaktorischen Suada gelangen zu können. Der Text wird derart nicht nur ",theoriegesteuert“ interpretiert. Sondern zweckdienlich kurzerhand umgeschrieben, also „theoriegesteuert" gelesen. Angesichts solcher circensischen Wissenschaftsauffassung überkommt einen das Kafka'sche Galeriebesucher-Syndrom, geschildert in Auf der Galerie im allerletzten Satz des Textes: „Da dies so ist, legt der Galeriebesucher das Gesicht auf die Brüstung und, im Schlussmarsch wie in einem schweren Traum versinkend, weint er, ohne es zu wissen." Ende der Vorstellung. 
Um es aufwendig-seriöser (und damit vielleicht neutral-allzu neutral), jedenfalls aber abschließend auszudrücken: „Der aufs Universelle, Menschliche und Grundlegende ausgerichteten phänomenologischen Wahrheitssage wird eine Diagnostik von Kehrtwendungen, Umstellungen und Andersverwendungen entgegengestellt. Darin steckt die Wahl eines Denkens, das keinen Anfang, keinen Ursprung und kein Fundament für sich in Anspruch nehmen kann, sondern seine Berechtigung der Analyse anonymer Funktionen und nicht der Auslegung evidenter Bedeutungen verdankt.“" ${ }^{35}$ Heinz Budes soeben zitierter Artikel über Derrida erwähnt freilich auch: „Aus der Richtung von Habermas wiederum ist Derrida das leichtsinnige Verspielen des Erbes der Aufklärung vorgeworfen worden." ${ }^{36}$ Das in diesen Zusammenhang gestellte Kafka-Beispiel (die „Legende“ Vor dem Gesetz) scheint also in diesem Zusammenhang ein sehr angemessenes zu sein. Denn über den Stellenwert von Gerechtigkeit und Recht in Derridas Denken hat wiederum Bude ausführen können: „Für Derrida scheint jedes System des Rechts auf den ebenso notwendigen wie gefährlichen Gedanken einer Gerechtigkeit ohne Recht zu beruhen. Derridas Blick vom Rand aus, der den Schwindel oder den Taumel nicht scheut, rechnet in Berufung auf Benjamin mit der Möglichkeit einer Gewalt, die jene dem Menschen unzugängliche Entscheidung fordert, Leben zu opfern, um das Lebendige zu retten“ - „woraus sich die Forderung einer Gabe ohne Austausch, ohne Kalkül, ohne Regel und ohne Rationalität ergibt.“37 Eine solche „Gabe ohne Austausch, Regel und Rationalität" stellt Jacques Derridas vorgestellter Vortrag über Kafkas Vor dem Gesetz, in seinem paradigmatischen Bemühen um „Dekonstruktion“ allerdings dar, eine wie berauscht wirkende Absage an die Forderungen jeder Logik als verbindlich gerade für die „Erste Kultur“, ein Akt im Zuge dessen, was dann Hans Magnus Enzensberger das „Kasperletheater der Dekonstruktion“ wird nennen können. Hier zeigte sich paradigmatisch, dass zwischen den Anforderungen einer zeitgemässen „Dritten Kultur“ und denen der heruntergegangenen Postmoderne (oder meinet halber auch „French Theory“) es keinerlei Vereinbarkeit zu geben vermag. Die angeführte Spielart der Kafka-Deutung erscheint als das Bermudadreieck aller philologischen Vernunft; ein (zu allem Überfluss auch noch französisches) Las Vegas der philologischen Belustigung, in dem es keine Leuchttürme mehr

\footnotetext{
${ }^{35}$ Heinz Bude, Erbschaft des schuldigen Denkens. In: Merkur, 59. Jg. (2005), H. 3, S. $1-96$.

${ }^{36}$ Heinz Bude, Erbschaft des schuldigen Denkens, a. a. O., S. 199.

${ }^{37}$ Heinz Bude, Erbschaft des schuldigen Denkens, a. a. O., S. 205.
} 
gab und auch nicht mehr geben sollte - sondern nur noch das blendende Feuerwerk amüsanter Beliebigkeit. Die Illuminationen waren, vielleicht, gut für's entertainment. In jedem Fall waren sie schlecht für die Orientierung. Sie blendeten, statt zu erhellen - vor allem, wenn man an's erhellende Feuer des altgriechischen Mythenhelden Prometheus denkt, dessen segensreicher Wärme wir uns nun zuwenden wollen. An dessen Leuchtkraft und lebensspendender Erleuchtung hat auch der allzu früh verstorbene Axel Gellhaus teil, dessen bahnbrechendem Essay über „Kafkas narrative Relativitätstheorie“ hier die Reverenz zu erweisen ist. ${ }^{38}$ Und auch das „Kafka-Handbuch“ des Metzler Verlags muss in diesem Kontext erwähnt werden. ${ }^{39}$

${ }^{38}$ Axel Gellhaus, ,Scheinbare Leere‘. Kafkas narrative Relativitätstheorie, in: Kopflandschaften - Landschaftsgänge, hrsg. v. A. Gellhaus, Christian Moser, Helmut J. Schneider, Köln, Weimar, Wien (Böhlau Verlag), 2007, S. 277 ff.

${ }^{39}$ Kafka-Handbuch, hrsg. v. Manfred Engel und Bernd Auerochs, Stuttgart, Weimar 2010. 
Open Access Dieses Kapitel wird unter der Creative Commons Namensnennung 4.0 International Lizenz (http://creativecommons.org/licenses/by/4.0/deed.de) veröffentlicht, welche die Nutzung, Vervielfältigung, Bearbeitung, Verbreitung und Wiedergabe in jeglichem Medium und Format erlaubt, sofern Sie den/die ursprünglichen Autor(en) und die Quelle ordnungsgemäß nennen, einen Link zur Creative Commons Lizenz beifügen und angeben, ob Änderungen vorgenommen wurden.

Die in diesem Kapitel enthaltenen Bilder und sonstiges Drittmaterial unterliegen ebenfalls der genannten Creative Commons Lizenz, sofern sich aus der Abbildungslegende nichts anderes ergibt. Sofern das betreffende Material nicht unter der genannten Creative Commons Lizenz steht und die betreffende Handlung nicht nach gesetzlichen Vorschriften erlaubt ist, ist für die oben aufgeführten Weiterverwendungen des Materials die Einwilligung des jeweiligen Rechteinhabers einzuholen.

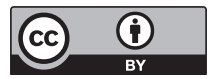

